\title{
Niveles de Presión Sonora en una Empresa de Maquinaria Pesada: Estrategias Administrativas y Técnicas para su Disminución
}

Sound pressure levels in a heavy machinery company: administrative and technical strategies for its reduction

\author{
Nancy Vega Gómez ${ }^{1}$, Elías A. Bedoya Marrugo ${ }^{1}$ Darío D. Sierra Calderón ${ }^{1}$ y Carlos \\ Severiche-Sierra ${ }^{1 *}$
}

\begin{abstract}
Resumen
Se diseñaron estrategias administrativas y técnicas para disminuir la exposición a niveles de presión sonora en una empresa de remolcadores. Realizándose visitas de campo y entrevistas, medición de niveles de presión sonora con sonómetro y dosímetros, formulación de estrategias administrativas y técnicas para control de exposición. Se detectaron niveles de presión sonora en área operativa que oscilan entre 101.4 decibeles a 104,4 decibeles, proyectado a 8 horas. Se determinaron los niveles de presión sonora a los que se encuentran expuestos 6 operadores de los cuartos de máquinas, a través de una sonometría y seis dosimetrías, estableciendo que el espectro del ruido existente en el cuarto de máquinas representa una exposición alta, superando los valores límites permisibles. A partir de estos resultados, se plantearon estrategias de mejora para la empresa y trabajadores, por medio de programas de capacitación y asesoramiento para la asignación de elementos de protección personal con índices de atenuación pertinentes al tipo y nivel de ruido existente en el área de trabajo y de un manual.

\section{Abstract}

Administrative and technical strategies were designed to reduce exposure to sound pressure levels in a tugboat company. Field visits and interviews, measurement of sound pressure levels with sound level meter and dosimeters, formulation of administrative strategies and techniques for exposure control. Sound pressure levels were detected in operating area ranging from 101.4 decibels to 104.4 decibels, projected at 8 hours. The sound pressure levels to which 6 engine room operators were exposed were determined through a sonometry and six dosimetry, establishing that the noise spectrum in the engine room represents a high exposure, exceeding the values Limits. Based on these results, improvement strategies were proposed for the company and workers, through training and counseling programs for the assignment of personal protection elements with attenuation indices pertinent to the type and level of noise existing in the work area, By means of a manual.
\end{abstract}

\section{Palabras Clave}

Límite permisible; medición; presión sonora; ruido.

Key words

Allowable limit; exposure; measurement; noise; sound pressure.

${ }^{1}$ Facultad de ingeniería, Fundación Universitaria Tecnológico Comfenalco, Cartagena de indias, Colombia.

*Autor correspondiente: cseveriches@gmail.com

Manuscrito recibido 20-02-2017; revisado 15-05-2017; aceptado 05-06-2017.

\section{Introducción}

Las empresas buscan conseguir sus metas en un mundo desarrollado por la innovación y la tecnología, por lo tanto, deben establecer políticas de control y mejora continua en sus procesos. En la empresa, el proceso en estudio es el relacionado con el nivel de presión sonora generado durante las operaciones de maquinado, lo cual genera problemas disconformidad en los trabajadores por la percepción a la alta presión sonora [1] durante las operaciones de maquinado; convirtiéndose esta situación en el objetivo central del trabajo de investigación, el cual se inicia por la caracterización de la operacionalización 
de dicho oficio, estableciendo tiempos máximos de exposición para los jefes de máquinas en sus respectivos puestos de trabajo [2]. Luego, se determina los actuales controles para ruido ocupacional durante las labores de maquinado, que permiten establecer el compromiso de la organización y de los trabajadores expuestos ante este peligro [3]. Posteriormente, se establecen los niveles de exposición a presión sonora durante las operaciones de maquinado, especificando su nivel de riesgo dentro de los límites permisibles [4], lo que permite formular las estrategias administrativas y técnicas que se evidencias en manual [5], que al ser aplicado en las organizaciones permitirá disminuir la exposición de los niveles de presión sonora en la empresa.

\section{Materiales y métodos}

La investigación es de tipo proyectivo [6], en el diseño de estrategias administrativas y técnicas; que permiten dar solución a un problema o necesidad de tipo práctico, en un área particular de trabajo, ocupándose de cómo deberían ser los sucesos, para alcanzar unos fines, dando funcionamiento adecuado al proceso de maquinado que da origen a la exposición de niveles elevados de presión sonora, siendo este el evento a modificar [7], identificando en las etapas analítica y explicativa; las causales a nivel técnico y administrativo en una empresa de remolcadores. Para obtener estos resultados se realiza una revisión documental, que incluye estudios sobre sonido, la exploración de normas técnicas de evaluación del ruido en la industria y los niveles permisibles de presión sonora [8]. Posteriormente, se realiza una visita de campo a la empresa con el fin de inspeccionar las áreas donde se desarrolla el trabajo investigativo [9], reconociendo las áreas con exposición a ruido e identificando los trabajadores expuestos a niveles de ruido ocupacional [10]. Con el apoyo de la revisión de los manuales de funciones de los jefes de máquinas en compañía de la Persona Designada en Tierra (DPA) [11], se realiza la caracterización de los puestos de trabajo; estableciendo los tiempos promedios de exposición a los niveles de presión sonora, a partir de la operacionalización del oficio de Jefe de máquinas. Luego, se aplican entrevistas de tipo formal [12]; al coordinador de seguridad y al personal operativo del área de maquinado con la finalidad de evidenciar el conocimiento que estos funcionarios poseen respecto a los controles implementados por la empresa frente al ruido ocupacional. Por otra parte, se utiliza el paquete estadístico T-lab versión 9.1.3 [13], en el que se analizan las entrevistas realizadas en la empresa. De igual modo, se requiere de la instrumentación acústica [14], que permita recolectar datos sobre los niveles de presión sonora en las áreas de maquinado [1], realizando seis dosimetrías a igual número de trabajadores (jefes de máquinas) y una sonometría para establecer el espectro de ruido en el área de cuarto de máquinas de un remolcador, realizando antes y después de cada medición la respectiva calibración de equipos, con el fin de garantizar la confiabilidad de los resultados obtenidos. Los equipos utilizados para tal fin fueron: dosímetros personales, sonómetro integrador de alta precisión y calibrador acústico. Los datos obtenidos se procesaron en el software SUITE PRO II, para obtener el espectro de ruido, los niveles pico y el nivel de presión sonora ponderado en el tiempo proyectado a ocho horas. Con el análisis de los resultados obtenidos se establecen las estrategias administrativas y técnicas consignadas en un manual, a consideración de la empresa, respecto a las acciones, que permiten tener un manejo adecuado de los niveles de presión acústica de las áreas en estudio.

\section{Resultados}

De la revisión documental realizada sobre estudios anteriores referentes a medidas de control de altos niveles de presión sonora; se establece la necesidad de cuantificar dichos niveles de presión acústica con la finalidad de atenuarlos [15], por medio del cálculo de la diferencia del nivel de presión sonora con y sin protector auditivo. Por otra parte, la publicación de un documento protocolo [16], establece la metodología y procedimientos necesarios para la evaluación, control y seguimiento del ruido provenientes de fuentes fijas instituyendo los niveles de emisión sonora máximos permisibles [17], además de plantear que la evaluación debe realizarse en condiciones reales de funcionamiento, en cuanto al horario del operario, espacio de trabajo y fuente sonora. De otro lado, el planteamiento de documentos guías y de procedimientos sobre la caracterización del ruido; permiten establecer los niveles de presión sonora, el espectro de frecuencias y el tipo de ruido, con la ayuda de instrumentación acústica, con el fin de realizar una comparación entre los resultados de las mediciones con los niveles permisibles para ruido [18]. De otro lado, los lugares de trabajo como embarcaciones [19]; deben disponer de ciertas características materiales y constructivas para que ningún trabajador esté expuesto a niveles de ruido superiores al valor límite establecido. Para lograrlo, se deben aplicar la normatividad que incluye pautas de actuación preventiva práctica y segura, debido a que las principales fuentes generadoras de ruido son los motores principales de la sala de máquinas, cuyos niveles de ruido dependen de la potencia y revoluciones por minuto. En la mayoría de los buques, el nivel de ruido puede oscilar entre $100-110 \mathrm{~dB}$, identificando los niveles de exposición a ruido, a través de la sonometría, siendo estos resultados mayores cuando el buque se encuentra en navegación, aunque se debe mencionar que las mediciones higiénicas de ruido a bordo de los buques de pesca no es control permanente en el sector. 

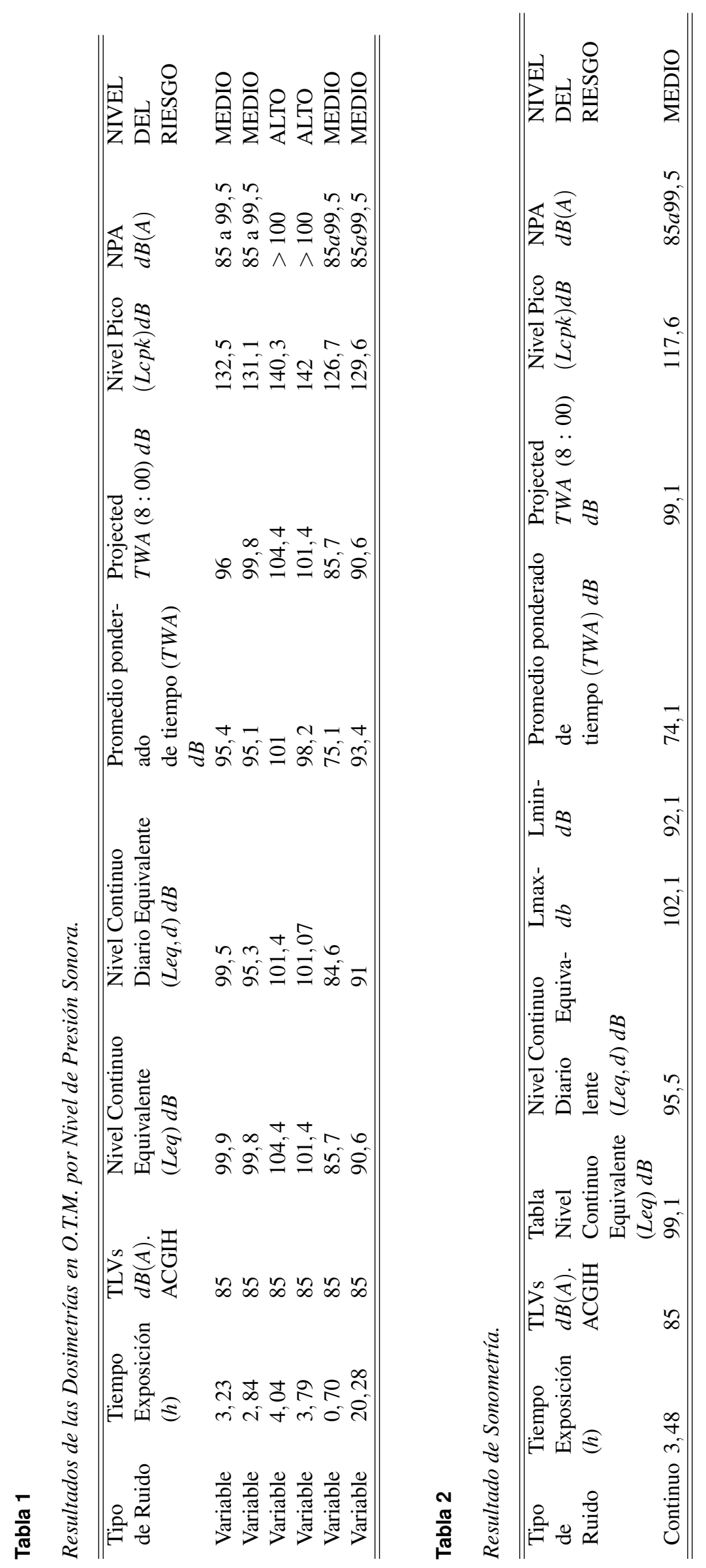


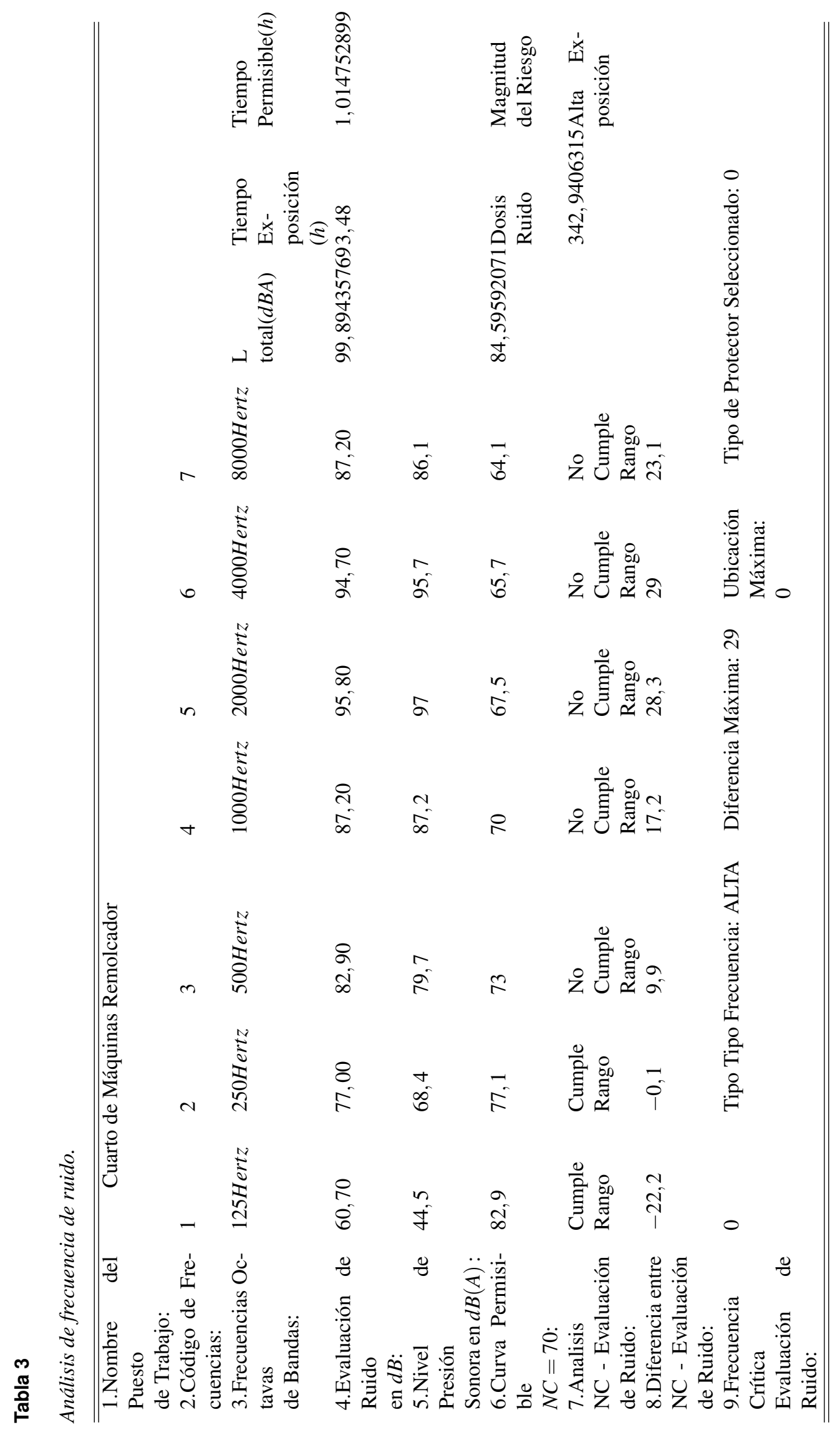


De otra parte, se debe mencionar, conceptos relevantes sobre el sonido, cuyo origen es la vibración de algún objeto, estableciendo a la vez una sucesión de ondas de compresión o expansión a través del medio que las soporta (aire, agua y otros) [20]. La unidad de intensidad del sonido es el Decibel (dB) y es medida por un sonómetro [21]. El sonido se puede definir en términos de las frecuencias que determinan su tono; refiriéndose a la sensación sonora entre grave y agudo, la intensidad; como la energía o potencia sonora para atravesar una superficie y calidad, junto con las amplitudes de las ondas sonoras [22]. La potencia sonora [20]; es la cantidad de energía acústica que se emite en una unidad de tiempo y se mide en vatio (w). La presión sonora [?]; es la cantidad de energía acústica por unidad de superficie $\left(\mathrm{N} / \mathrm{m}^{2}\right)$. El margen de presión acústica que es capaz de oír una persona joven y normal, oscila entre $20 \mathrm{~N} / \mathrm{m}^{2}$ y $2 \times 10-5 \mathrm{~N} / \mathrm{m}^{2}$, denominándose a esto umbral auditivo. La intensidad sonora [23]; es la cantidad de energía acústica que pasa a través de la unidad de superficie perpendicular a la dirección de propagación por unidad de tiempo y se expresa en $\left(\mathrm{W} / \mathrm{m}^{2}\right)$. Las frecuencias audibles para el oído humano van de 20 a 20.000 ciclos por segundo o Hertz $(\mathrm{Hz})$. De $0 \mathrm{~Hz}$ a $20 \mathrm{~Hz}$ son infrasonidos y más de $20.000 \mathrm{~Hz}$ son ultrasonidos [24]. El Sonido es percibido por una persona cuando el elemento sonoro incide en el oído [?], desencadenando el proceso de percepción a través del oído externo (receptor de ondas), oído medio (amplificador) y oído interno (integración e interpretación de señales acústicas) [25].

Es relevante mencionar que la American Conference of Governmental Industrial Hygienists (ACGIH) [26]; aporta el establecimiento de los valores límites permisibles o "Threshold Limit Values" (TLV); basados en criterios científicos de protección de la salud [27]. Estos TLV; son límites recomendados de niveles de presión sonora por tiempo de exposición, a fin de priorizar el riesgo inherente [28].

El objetivo de este estudio es diseñar estrategias administrativas y técnicas que permitan controlar los altos niveles de presión sonora durante las operaciones de maquinado en remolcadores, para tal fin se desarrollan las siguientes actividades. Para la caracterización del oficio de Jefes de Máquinas [29], se determina un tiempo promedio máximo de exposición de 22 minutos por maniobra; durante la actividad de Dirección y Control de Máquinas [28]; realizando tareas como: encendido de generador, tablero eléctrico, bombas, compresores, sistema neumático, motor principal, bombas de timón. Revisión de sistemas de: temperatura, refrigeración, combustible, enfriamiento.

Igualmente, de las entrevistas, se destaca que a los jefes de máquinas entrevistados les falta claridad sobre los controles dispuestos por la empresa para minimizar la exposición a ruido ocupacional, y los tiempos para la realización de sus exámenes médicos ocupacionales [15]. Respecto al programa de capacitación, conocen las diferentes temáticas, faltando regularizar la programación. Los jefes de máquinas, tienen claras sus funciones respecto al cargo. Respecto a los con- troles a nivel de fuente, no pueden llevarse a cabo debido a la reglamentación dada por la Asociación Internacional de Sociedades de Clasificación (IACS) [30], sosteniendo que se debe mantener el esbozo y la estructura de la embarcación desde su diseño hasta el fin de su vida útil [31]. Por último, falta claridad por parte de los jefes de máquinas entrevistados, en el reconocimiento de la Aseguradora de Riesgos Laborales (ARL), suscrita por la empresa, ni los aportes que dicho organismo hace respecto a capacitación, acompañamiento, vigilancia y control del riesgo a ruido ocupacional. Por otra parte, de estas mismas entrevistas [12], se obtienen resultados como: asociación de 60 palabras distribuidas en un plano cartesiano por grupos según: las condiciones actuales de la población trabajadora, los aspectos actuales de sitio de trabajo, las tendencias negativas del proceso de trabajo y los hechos positivos y negativos con tendencia amenazante a ser aspectos negativos del actual sitio de trabajo.

De igual forma, se establece el análisis de correspondencia, por ocupación, en donde el Coordinador SISO, plantea la relación entre las palabras: seguridad, trabajador, implementación, personas, autocuidado, temas y riesgo. Mientras que el aprendiz y un jefe de máquinas, asocian las palabras: presentar, accidente, responder, entregar y capacitación. Por último, un Jefe de Máquinas relaciona las palabras: examen, ruido, empresa, ingresar, protector, EPP, manejo y uso. Por otro lado, se realiza el análisis de correspondencia según el tiempo de antigüedad en la empresa, mostrando la asociación que los trabajadores con menos de un año en la empresa tienen respecto a las palabras: máquina, capacitación, entregar, responder, presentar y accidente. La asociación hecha por los trabajadores entre 1 y 2 años es con las palabras: manejo, uso, EPP, empresa, ruido, examen, protector e ingresar y la asociación de palabras elaborada por los trabajadores con más de 2 años de trabajo, con las palabras: trabajador, seguridad, autocuidado, temas, riesgo, personal e implementación. Por último, no hay relación de las palabras: trabajar, realizar, mantenimiento y mes.

De otro lado, también se realiza la interpretación de los resultados de las mediciones de los niveles de presión sonora, con base en lo dispuesto por la ACGIH, con un límite permisible de $85 d B$ para una jornada laboral de 8 horas al día, usando una tasa de intercambio de $3 d B$, además de un nivel de acción de $80 d B(A)$. De los resultados de las emisiones respecto a los límites permisibles [11], se obtuvo la evaluación de la exposición al ruido de los operarios, a través de dosimetrías; determinando que existe una exposición a ruido de tipo variable; debido a las distintas tareas que los Jefes de Máquinas realizan en la operación de los remolcadores en los cuartos de máquinas. Las dosis obtenidas en los cuartos de máquina de los remolcadores, sobrepasada el acuerdo al promedio ponderado en el tiempo de 8 horas de trabajo, siendo el resultado más crítico el obtenido durante maniobra en un remolcador; es de $101,4 d B$ y $104,4 d B$; siendo este un nivel de riesgo ALTO. Mientras que para los demás remolcadores es MEDIO, con niveles entre $85,7 d B$ y $99,8 d B$, tal como lo muestran los 
resultados en la Tabla 1.

Para el caso de la sonometría [32], el tipo de ruido generado es de tipo continuo. El remolcador se encontraba en plataforma en el momento de realizar la sonometría, y su resultado con una proyección a 8 horas del promedio ponderado en el tiempo; es de 99, $1 d B$ como se muestra en la Tabla 2; superando el criterio de referencia de la ACGIH, siendo el límite permisible de $85 d B(A)$ para una jornada laboral de 8 horas, con una tasa de intercambio de $3 d B(A)$, dando un Nivel de Riesgo Medio.

Con el análisis de frecuencias del ruido generado en las máquinas del Remolcador como se evidencia en la Tabla 3, no se cumple con los criterios de Puntuación de ruido (NR) de la Organización Internacional de Estandarización (ISO R-1996) [33] en las frecuencias bajas, medianas y altas. La frecuencia más crítica es la de $2000 \mathrm{~Hz}$ (alta); con $27,2 \mathrm{~dB}$ más con relación al nivel de energía $67,5 d B$ correspondiente al nivel de reducción de $70 d B$. Por último, de acuerdo a las distintas tareas del operario, se recomienda establecer el control de ruido en la selección del protector auditivo, con el criterio de reducción " $\mathrm{R}$ " de $70 \mathrm{~dB}$ con el fin de garantizar la prevención de la sordera profesional en forma eficaz [27].

\section{Discusión}

Determinar en primera medida la caracterización del oficio de jefes de máquinas, permite establecer los tiempos máximos de exposición de niveles de presión sonora, que dicho personal posee durante la ejecución de sus actividades durante cada una de las maniobras a realizar [7], en el transcurso de su jornada laboral.

La realización de las mediciones acústicas [20], por medio de sonometrías y dosimetrías; permite acceder tanto a la identificación de los actuales Niveles de Presión Sonora como del tipo de ruido ocupacional; siendo en este caso un tipo de ruido variable y que supera los límites permitidos por la ACGIH, por lo que el Nivel de Riesgo para la población trabajadores de jefes de máquinas se encuentra entre Medio y Alto; durante su jornada laboral. Esto permite, establecer el punto de partida para la formulación de las medidas pertinentes para minimizar la exposición al riesgo ocupacional a ruido por parte de los trabajadores.

Por otra parte, es relevante mencionar, que toda empresa debe contar con la gestión de cada uno de sus riesgos. En la actual situación de la empresa; a pesar del trabajo desarrollado por parte de la Coordinación SISO, para mantener la salud integral de sus empleados, se evidencia que aún los jefes de máquinas no poseen una identificación clara tanto de los controles administrativos como de los técnicos aplicados por parte de la empresa, a fin de preservar su salud auditiva. Tal vez sea necesario que la empresa gestione otro tipo de estrategias más contundentes al fortalecimiento y apropiación de este tipo de temáticas vitales para la salud de su población trabajadora, afianzando dichos tópicos con un seguimiento y control permanente.
Además, es importante resaltar que, aunque las casas clasificadoras [31], no permiten el cambio en el diseño de nuevas instalaciones, incorporación de nuevos procesos, ampliación y modificación de los existentes, se debe considerar otros criterios preventivos durante la planeación de futuros diseños de remolcadores, a fin de que estos permitan mitigar o controlar el ruido. Algunas de las medidas a implementar pueden ser: mejoras en la ubicación y disposición de los puestos de trabajo, mejoras en las fuentes generadoras de ruido; a través de la consideración de aspectos arquitectónicos y constructivos del área de trabajo [31] como por ejemplo instalación de materiales aislantes de ruido y vibraciones, así como el encerramiento de maquinaria generadora de altos niveles de presión sonora. De otro lado, es fundamental que la empresa cuente con la información del fabricante de cada uno de los equipos y maquinaria dispuesta en el cuarto máquinas de los remolcadores; con el fin de conocer los niveles de emisión de ruido de la fuente sonora (propulsores y generados) [19], para consideraciones previas y futuras en el control de ruido, establecidos a través de la matriz de riesgo elaborada por la empresa. De igual forma, la elaboración de los procedimientos respecto del uso correcto al uso de las máquinas y su mantenimiento [10]; deben considerarse de acuerdo a las instrucciones dadas por el fabricante, lo que permitirá gestionar un plan de capacitación para el trabajador, a través de un equipo de trabajo interdisciplinario.

También, es indispensable mencionar que la exposición ocupacional a ruido ocupacional en este tipo de lugares de trabajo (cuarto de máquinas de remolcadores), debe ser un proceso dinámico, es decir, no debe ser abordado una sola vez, por lo que se deben considerar criterios de periodicidad determinados por la misma empresa [14]. Los resultados obtenidos en la evaluación inicial de la exposición ocupacional a ruido ocupacional, deben ser comparados con los Criterios de Acción [11], según corresponda al tiempo de exposición, con el fin de establecer las medidas de control del ruido. De igual forma, es pertinente que se realicen reevaluaciones de la exposición al ruido ocupacional para verificar la efectividad de las medidas de control implementadas. Cada vez que dichos resultados superen los límites permisibles, la empresa deberá efectuar nuevos mecanismos de control, con el fin de establecer y garantizar ambientes de trabajo seguros, es decir que la exposición al ruido ocupacional logre estar siempre por debajo de los límites permisibles.

No obstante, las medidas de control a implementar, deben empezar desde la fuente u origen. De no ser factible, se deben implementar medidas en el medio de propagación, y en última instancia, se deben considerar las medidas de control en el receptor (trabajador). La empresa, se encuentra actualmente vinculada a la normatividad establecida por la IACS; por lo que dichos controles en los cuartos de máquinas de los remolcadores, solamente se aplican a los trabajadores, de tal forma, que continuamente se refuercen estos controles, a fin de afianzar el compromiso de los trabajadores con las intervenciones realizadas por parte de la empresa. 
Para el caso en estudio, entre los controles realizados por la empresa a nivel técnico, se encuentran el diseño e instalación de cabinas que minimizan y obstaculizan la trayectoria de la onda sonora en dos de los remolcadores de la empresa. Con respecto a los controles administrativos, la empresa posee: programa de capacitación, programa de mantenimiento preventivo y correctivo, programa de vigilancia epidemiológica, programa de elementos de protección personal (protección auditiva), señalización y advertencia de lugares de trabajo ruidosos y el acompañamiento por parte de la Administradora de Riesgos Laborales (ARL).

\section{Conclusiones}

Los resultados presentados ratifican la importancia de los controles administrativos y técnicos para el adecuado mantenimiento de la salud auditiva de los trabajadores expuestos a ruido ocupacional. Por otra parte estos proporcionan una valiosa evidencia del fortalecimiento de trabajo en equipo y del compromiso que deben asumir los directivos y el personal operativo de las organizaciones, con el fin de conseguir los cambios deseados en los comportamientos de todo el grupo empresarial, logrado a través de programas de capacitación, de vigilancia y control epidemiológico, regularidad de las mediciones acústicas y en general todo proceso que conlleve al mantenimiento de la salud integral de la población trabajadora.

\section{References}

[1] J. C. M., J. E. R., H. F. G., M. M. I., M. M. J., R. M. noz A., A. Pérez, L. R. J., A. M. S. B., M. S. V., and J. C. V. I, "Guía perventiva para los trabajadores expuestos a ruido," tech. rep., Instituto de Salud Pública de Chile, 2012.

[2] R. Sibaja, Salud Y Seguridad en El Trabajo. EUNED, 2002.

[3] C. nacional del medio ambiente, "Norma de emisión de ruidos molestos generados por fuentes fijas," 2000.

[4] J. Cortés and J. Díaz, Técnicas de prevención de riesgos laborales: seguridad e higiene del trabajo. Tébar, 2007.

[5] D. de Planeación, "Guía técnica para la elaboración de manuales de procedimientos," techreport, Instituto Politécnico Nacional, 2014.

[6] J. H. de Barrera, Cómo Formular Objetivos de Investigación: Un acercamiento desde la Investigación Holística. 2012.

[7] A. Hernández Díaz and B. M. González Méndez, “Alteraciones auditivas en trabajadores expuestos al ruido industrial," Medicina y Seguridad del Trabajo, vol. 53, pp. 09 - 19, 092007.

[8] R. Osío Cabrices, "Ruido: el enemigo invisible," Debates IESA, vol. XVII, pp. 72-75, Oct. 2012.

[9] O. M. Internacional, EVALUACIÓN A BORDO, Edición de 2001. IMO Publishing.
[10] M. Fernández-Ríos, Análisis y descripción de puestos de trabajo: teoría, métodos y ejercicios. Díaz de Santos S.A., 2004.

[11] J. Espinosa, L. Carriel, M. Sánchez, J. C. Valenzuela, H. Fontecilla, R. Concha, L. Molina, M. Parra, V. Nehgne, C. Tapia, J. C. Miranda, N. Pizarro, and J. P. na, "Protocolo sobre normas mÍnimas para el desarrollo de programas de vigilancia de la pÉrdida auditiva por exposición a ruido en los lugares de trabajo."

[12] R. E. L. Estrada and J.-P. Deslauriers, "La entrevista cualitativa como técnica para la investigación en trabajo social," Margen, pp. 1-19, June 2011.

[13] J. W. Sammon, "A nonlinear mapping for data structure analysis," IEEE Transactions on Computers, vol. C-18, pp. 401-409, May 1969.

[14] A. Positiva, "Programa de vigilancia epidemiológica para la conservación auditiva," Sept. 2010.

[15] D. S. Rodriguez and C. S. Maldonado, "Programa de capacitación en seguridad y salud en el trabajo, basado en los factores de riesgo laborales, de las actividades de belleza en el sector informal del barrio san cristobal norte," techreport, Universidad Militar Nueva Granada, 2014.

[16] C. A. E. L. no and A. E. G. Fernández, "Protocolo para medir la emisión de ruido generado por fuentes fijas," Revista Ingenierías Universidad de Medellín, vol. 10, no. 18, pp. 51-59, 2011.

[17] P. Floría and F. C. (Madrid), Gestión de la higiene industrial en la empresa. Fundación Confemetal, 2007.

[18] A. E. para la Calidad, "Mantenimiento," 2015.

[19] S. T. S. D. de Desarrollo de Proyectos e Innovación., "Estudio del impacto del ruido en la salud de los trabajadores del mar."

[20] M. Mancera, "El ruido y su evaluación. seguridad y salud en el trabajo," 2013.

[21] A. Sura, 2015.

[22] A. Ruiz, P. Floría, and D. Maestre, Manual para el técnico en prevención de riesgos laborales: nivel básico. Fundación Confemetal, 2006.

[23] P. Tipler and G. Mosca, Física para la ciencia y la tecnología. No. v. 1 in Física para la ciencia y la tecnología, Reverté, 2005.

[24] U. P. de Valencia, “Tecnologías de la imagen 2," 2002.

[25] M. G. Martínez, J. J. J. Garcí, Y. L. Ceballos, A. M. Valencia, M. A. V. Zapata, and E. M. V. T, "Ruido industrial: Efectos en la salud de los trabajadores expuestos," CES Salud Pública, vol. 3, no. 2, pp. 174-183.

[26] ACGIH, "Defining the science of occupational and environmental health," in Proceedings American Conference of Governmental Industrial Hygientists, 2015. 
[27] P. L. Mendaza and J. G. Farrás, "Estimación de la atenuación efectiva de los protectores auditivos," 2003.

[28] M. de Trabajo y Seguridad Social, "Sistema general de riesgos profesionales. reglamento técnico para exposición a ruido," 2000.

${ }^{[29]}$ M. y Gerencia, "Las sociedades de clasificación de buques," 2015.

[30] IACS, 2015.

[31] C. de conocimiento de la industria marítima, 2015.

[32] B. Espinosa, "Guía para la selección y control de protecctores auditivos," tech. rep., Universidad Austral de Chile, 2006.

[33] C. R. PLC, "Calculation of nr \& nc curves in the optimus sound level meter and the noisetools software," techreport 31, 2013. 